\title{
A Generalized Circle Theorem on Zeros of Partition Function at Asymmetric First Order Transitions
}

\author{
Koo-Chul Lee \\ Department of physics and the Center for Theoretical Physics \\ Seoul National University \\ Seoul, 151-742, Korea
}

(October 26, 2018)

\begin{abstract}
We present a generalized circle theorem which includes the Lee-Yang theorem for symmetric transitions as a special case. It is found that zeros of the partition function can be written in terms of discontinuities in the derivatives of the free energy. For asymmetric transitions, the locus of the zeros is tangent to the unit circle at the positive real axis in the thermodynamic limit. For finite-size systems, they lie off the unit circle if the partition functions of the two phases are added up with unequal prefactors. This conclusion is substantiated by explicit calculation of zeros of the partition function for the Blume-Capel model near and at the triple line at low temperatures.

05.50.+q, 05.70.Fh, 64.60.-i, 75.10.Hk
\end{abstract}

Typeset using REVTEX 
More than three decades have passed since Yang and Lee [1] first published their celebrated papers on the theory of phase transitions and the circle theorem on the zeros of the partition function of ferromagnetic Ising models. Although there have been some developments in extending the theorem [2], little is known about what happens to the theorem in the case of more general first-order transitions such as asymmetric transitions or temperature driven transitions. In their original proof of the theorem, Lee and Yang relied on particular properties of the coefficients of the polynomial. Furthermore they used an analogue of a certain electrostatic problem to relate the discontinuity in the spontaneous magnetization to the density of zeroes. In this paper we present a simple proof of the circle theorem which can be extended to first-order phase transitions of the most general kind. The extended theorem can be used to (1) identify the order of the phase transition, since a second-order phase transition manifests drastically different characteristics in the complex partition function [3]; (2) pinpoint the transition point very accurately; and (3) resolve the recent controversy over the equal weight versus equal height of the probability distribution function at an asymmetric transition [4] among other potential applications.

The generalized theorem is based on a very simple property that the probability distribution for the order parameter $M$, conjugate to an external ordering field $H$ is doubly peaked in the two-phase region. This simple property alone is sufficient to prove the generalized circle theorem which states that the locus of zeros of partition function in the complex $e^{H_{-}}$ plane forms a circle near the positive real axis. Near a triple line where three phases coexist, there are three peaks in the distribution function, and this leads to some interesting behavior of loci of complex zeros. These conclusions are substantiated by explicitly calculating zeros of the partition function of the Blume-Capel model near and at the triple line at low temperatures.

Let us consider a partition function $Z(H)$ of a discrete lattice system made of a $d$ dimensional cube of side $L$ as a function of variable $H$; we seek the complex zeros of $Z(H)$. $H$ can be an external magnetic field for a spin system in which case the conjugate order parameter would be the magnetization. 
In the following we will consider only three phase coexistence for the sake of simplicity. Generalization to coexistence of more than three phases is straightforward if cumbersome. If we label the three phases as $A, B$ and $C$ and the partition function of each phase as $Z_{\lambda}(H)$, where $\lambda=A, B, C$, then we have near a triple point

$$
Z(H)=\sum_{\lambda} Z_{\lambda}(H)
$$

The fact that the partition function is made of three parts follows from the fact that there are two barriers, say at $K_{1}$ and $K_{2}$ in the order parameter $M$, which separate three phases. Therefore we can define $Z_{A}(h)=\sum_{M=M_{\min }}^{M=K_{1}} e^{h M} Z_{M}, Z_{B}(h)=\sum_{M>K_{1}}^{M=K_{2}} e^{h M} Z_{M}$ and $Z_{C}(h)=\sum_{M>K_{2}}^{M=M_{\max }} e^{h M} Z_{M}$, with $h=H / K_{B} T \equiv \beta H . Z_{M}$ is a partition function for fixed $M$ in case $H$ is the external field and we may take $\Omega(E)$, the number of configurations at energy $E$, when $H=T$ and $M=E$ for temperature driven transitions. We can always make $h=0$ at the coexistence point by redefining $Z_{M}$ appropriately.

Let $\bar{M}$ be $\max \left(\left|M_{\min }\right|,\left|M_{\max }\right|\right)$. Introducing the probability distribution function $p(x)=Z_{M=x \bar{M}} / Z(H=0)$, we can define the moment generating function $\mathcal{M}(t)=\left\langle e^{t x}\right\rangle \equiv$ $\sum_{x} e^{t x} p(x)=\sum_{l=0}^{\infty}\left\langle x^{l}\right\rangle t^{l} / l !$. Since $\left\|\left\langle x^{l}\right\rangle\right\| \leq 1, \mathcal{M}(t)$ is analytic in the whole complex $t$-plane. Since $\mathcal{M}(t=h \bar{M})=Z(h) / Z(0)$, zeros of partition function $Z(h)$ can be obtained from zeros of $\mathcal{M}(t)$. By separating the contribution from three phases, we can write $\mathcal{M}(t)=\mathcal{M}_{A}(t)+\mathcal{M}_{B}(t)+\mathcal{M}_{C}(t)$, where $\mathcal{M}_{\lambda}(t)=Z_{\lambda}(h=t / \bar{M}) / Z(0)$.

We first consider the case where only two phases $A$ and $C$ coexist. Introducing the cumulant generating function defined by $\psi_{\lambda}(t)=\ln \left(\mathcal{M}_{\lambda}(t)\right)$, we can rewrite $\mathcal{M}(t)=$ $2 e^{\bar{\psi}(t)} \cosh (\tilde{\psi}(t))$, where $\bar{\psi}=\left(\psi_{C}+\psi_{A}\right) / 2$ and $\tilde{\psi}=\left(\psi_{C}-\psi_{A}\right) / 2$. Zeros of the $\mathcal{M}(t)$ in the complex $t$-plane are simply solutions of following equation, $\tilde{\psi}\left(t_{k}\right)= \pm i(1 / 2+k) \pi$, where $k=0,1,2,3 \cdots$. Zeros of partition function in the complex $h_{k}$ can be calculated from the relation $h_{k}=t_{k} / \bar{M}$.

In order to express the zeros of $Z(h)$, in terms of the free energy density and its derivatives with respect to $h$ of each phase, we expand $\psi_{\lambda}$ in Taylor series of $h$ as $\psi_{\lambda}=\ln \left(\mathcal{M}_{\lambda}(0)\right)+$ $L^{d} \sum_{l=1}^{\infty} h^{l} \gamma_{l}^{\lambda} / l$ !, where $\gamma_{l}^{\lambda}=L^{-d} \partial^{l} \ln \left(Z_{\lambda}(h)\right) /\left.\partial h^{l}\right|_{h=0}$. Therefore $\tilde{\psi}(t=h \bar{M})=-\ln (a) / 2+$ 
$\tilde{F}(h)$, where $a=\mathcal{M}_{A}(0) / \mathcal{M}_{C}(0)$ and $\tilde{F}(h)=L^{d} \sum_{l=1}^{\infty} h l \tilde{\gamma}_{l} / l$ ! with $\tilde{\gamma}_{l}=\left(\gamma_{l}^{C}-\gamma_{l}^{A}\right) / 2$.

Therefore zeros of $Z(h)$ are solutions of the following equations,

$$
\begin{gathered}
\Im(\tilde{F}(h))= \pm(1 / 2+k) \pi \equiv I_{k} \\
\Re(\tilde{F}(h))=\ln (a) / 2 \equiv R .
\end{gathered}
$$

We can easily obtain zeros $Z(h)$ by inverting the series. Following the tradition, we will consider zeros in the $z$-plane defined by $z=e^{h}$. The modulus $r=|z|=e^{\Re(h)}$ and argument $\theta=\ln (z / r) / i=\operatorname{Im}(h)$ of zeros can be found from

$$
\begin{aligned}
& \ln \left(r_{k}\right)=\Re\left(h_{k}\right)=\hat{R}+\hat{\gamma}_{2}\left(-\hat{R}^{2}+\hat{I}_{k}^{2}\right)+\left(2 \hat{\gamma}_{2}^{2}-\hat{\gamma}_{3}\right) \\
& \times\left(\hat{R}^{3}-3 \hat{R} \hat{I}_{k}^{2}\right)+\left(-5 \hat{\gamma}_{2}^{3}+5 \hat{\gamma}_{2} \hat{\gamma}_{3}-\hat{\gamma}_{4}\right)\left(\hat{R}^{4}-6 \hat{R}^{2} \hat{I}_{k}^{2}+\hat{I}_{k}^{4}\right) \\
& +\left(14 \hat{\gamma}_{2}^{4}-21 \hat{\gamma}_{2}^{2} \hat{\gamma}_{3}+3 \hat{\gamma}_{3}^{2}+6 \hat{\gamma}_{2} \hat{\gamma}_{4}-\hat{\gamma}_{5}\right)\left(\hat{R}^{5}-10 \hat{R}^{3} \hat{I}_{k}^{2}\right. \\
& \left.+5 \hat{R} \hat{I}_{k}^{4}\right)+\left(42 \hat{\gamma}_{2}^{5}-84 \hat{\gamma}_{2}^{3} \hat{\gamma}_{3}+28 \hat{\gamma}_{2} \hat{\gamma}_{3}^{2}+28 \hat{\gamma}_{2}^{2} \hat{\gamma}_{4}-7 \hat{\gamma}_{3} \hat{\gamma}_{4}\right. \\
& \left.-7 \hat{\gamma}_{2} \hat{\gamma}_{5}+\hat{\gamma}_{6}\right)\left(-\hat{R}^{6}+15 \hat{R}^{4} \hat{I}_{k}^{2}-15 \hat{R}^{2} \hat{I}_{k}^{4}+\hat{I}_{k}^{6}\right) \ldots \\
& \theta_{k}=\Im\left(h_{k}\right)=\hat{I}_{k}-2 \hat{\gamma}_{2} \hat{R} \hat{I}_{k}+\left(2 \hat{\gamma}_{2}^{2}-\hat{\gamma}_{3}\right)\left(3 \hat{R}^{2} \hat{I}_{k}-\hat{I}_{k}^{3}\right) \\
& +4\left(5 \hat{\gamma}_{2}^{3}-5 \hat{\gamma}_{2} \hat{\gamma}_{3}+\hat{\gamma}_{4}\right)\left(-\hat{R}^{3} \hat{I}_{k}+\hat{R} \hat{I}_{k}^{3}\right)+\left(14 \hat{\gamma}_{2}^{4}-21 \hat{\gamma}_{2}^{2} \hat{\gamma}_{3}\right. \\
& \left.+3 \hat{\gamma}_{3}^{2}+6 \hat{\gamma}_{2} \hat{\gamma}_{4}-\hat{\gamma}_{5}\right)\left(5 \hat{R}^{4} \hat{I}_{k}-10 \hat{R}^{2} \hat{I}_{k}^{3}+\hat{I}_{k}^{5}\right) \\
& +\left(-42 \hat{\gamma}_{2}^{5}+84 \hat{\gamma}_{2}^{3} \hat{\gamma}_{3}-28 \hat{\gamma}_{2} \hat{\gamma}_{3}^{2}-28 \hat{\gamma}_{2}^{2} \hat{\gamma}_{4}+7 \hat{\gamma}_{3} \hat{\gamma}_{4}+7 \hat{\gamma}_{2} \hat{\gamma}_{5}\right. \\
& \left.-\hat{\gamma}_{6}\right)\left(6 \hat{R}^{5} \hat{I}_{k}-20 \hat{R}^{3} \hat{I}_{k}^{3}+6 \hat{R} \hat{I}_{k}^{5}\right) \cdots
\end{aligned}
$$

where $\hat{R} \equiv R / L^{d} \mu, \hat{I}_{k} \equiv I_{k} / L^{d} \mu, \hat{\gamma}_{l} \equiv \tilde{\gamma}_{l} / \mu l$ ! and $\mu \equiv \tilde{\gamma}_{1}$, is one half of the discontinuity of the order parameter across the phase boundary.

The angular density of the zeros, $g(\theta)$ defined by $L^{d} g(\theta)=1 /\left(\theta_{k+1}-\theta_{k}\right)$, can be obtained by differentiating the left hand side of (2) with respect to $\theta_{k}$. We have

$$
2 \pi g(\theta)=2 \tilde{\gamma}_{1}-\tilde{\gamma}_{3} \theta^{2}+\tilde{\gamma}_{5} \theta^{4} / 12-\cdots+O\left(L^{-d}\right)
$$

In the scaling limit, we have $r=\exp \left[\ln (a) /\left(2 L^{d} \mu\right)+O\left(L^{-2 d}\right)\right]$ and $\theta_{k}= \pm \pi(1 / 2+k) /\left(L^{d} \mu\right)+$ $O\left(L^{-2 d}\right)$. This implies that the zeros are distributed uniformly on a circle of varying radius 
near the positive real axis for finite system if the partition functions of the two phases are added up with unequal prefactors. If the prefactors are equal, i.e, $a=1$, then zeros form a circle of unit radius near the positive real axis in the scaling limit. However if the argument , $\theta_{k}$ grows large enough zeros deviate from the unit circle unless all $\tilde{\gamma}_{l}$ 's with even $l$ vanish. However for symmetric transitions we have not only $a=1$ but also $\tilde{\gamma}_{l}=0$ for all even $l$. Therefore we have $r_{k}=1$ for all sizes and the angular distribution of the zeros becomes exactly Eq. (6) without the size dependent correction terms. This is the Lee-Yang theorem. We now have the angular density of zeros in a complete power series in $\theta$.

Let us now consider a case where three phases coexist. Using $F_{B}(h)$ and $b$ defined by $F_{B}(h)=L^{d} \sum_{l=1}^{\infty} h^{l} \gamma_{l}^{B} / l !$ and $b=\mathcal{M}_{B}(0) / \mathcal{M}_{C}(0)$, we can modify eqs.(2) and (3) as

$$
\begin{array}{r}
e^{\Re(\bar{F}(h))}\left(e^{\Re(\tilde{F}(h))}+a e^{-\Re(\tilde{F}(h))}\right) \cos (\Im \tilde{F}(h)) \\
=-b e^{\Re\left(F_{B}(h)\right)} \cos \left(\Im\left(F_{B}(h)\right)\right), \\
e^{\Re(\bar{F}(h))}\left(e^{\Re(\tilde{F}(h))}-a e^{-\Re(\tilde{F}(h))}\right) \sin (\Im(\tilde{F}(h)) \\
=-b e^{\Re\left(F_{B}(h)\right)} \sin \left(\Im\left(F_{B}(h)\right)\right),
\end{array}
$$

where $\bar{F}(h)=\bar{\psi}(t=h \bar{M})$. General solution to the above equation is complicated if not impossible to obtain. Therefore we will consider a special case which is useful in analyzing the ensuing example. We will consider the case where $a$ and $c$ phases are symmetric and $b$ phase is also symmetric with respect to the midpoint between the two. Furthermore we will consider the scaling limit where corrections in $O\left(L^{-2 d}\right)$ is neglected. In that case we have

$$
\begin{array}{r}
\cosh (\Re(\tilde{F}(h))) \cos (\Im(\tilde{F}(h)))=-b / 2, \\
\sinh (\Re(\tilde{F}(h))) \sin (\Im(\tilde{F}(h)))=0 .
\end{array}
$$

If $b / 2 \leq 1$, we have zeros as $\Im(h)=\cos ^{-1}(-b / 2) / L^{d} \mu$ and $\Re(h)=0$ while for $b / 2>1$, $\Im(h)=(\pi(1+2 k)) / L^{d} \mu$ and $\Re(h)= \pm\left|\cosh ^{-1} b / 2\right| / L^{d} \mu$. This means that crossing the triple point from two phase region $(b / 2 \leq 1)$ to the single phase region $(b / 2>1)$, zeros on the unit circle disappear. Only for small sized systems and close to the triple point, we see 
two circles separating out from the unit circle which are sort of ghost circles that separate three phases, two unstable symmetric and the new stable phases.

We have tested the above conclusion explicitly by calculating zeros of the partition function of the Blume-Capel model. Consider the energy of the Blume-Capel model [7,8], $E=-H \sum_{i} S_{i}+D \sum_{i} S_{i}^{2}-J \sum_{<i, j>} S_{i} S_{j}$ of $N$ spins on a square lattice of side $L\left(N=L^{2}\right)$ on a torus. $H, D$ and $J$ are usual external parameters and $S_{i}$ are spin variables which take three values, \pm 1 and 0 . If we designate by $N_{+}$and $N_{-}$the number of +1 spins and -1 spins, and $N_{e} \equiv\left[(q / 2) \sum_{i} S_{i}^{2}-\sum_{<i, j>} S_{i} S_{j}\right]$, we can write the energy $E=-H\left(N_{+}-N_{-}\right)+$ $\Delta\left(\left(N_{+}+N_{-}\right)+J N_{e}\right.$ where $q$ is the coordination number and $\Delta \equiv D-J q / 2$. We have defined energy $N_{e}$ such a way to make the ground state a triple point, in the absence of external fields $H$ and $\Delta$. The partition function can be calculated according to

$$
Z(T, H, \Delta)=\sum_{N_{e}, N_{+}, N_{-}} e^{-E\left(N_{e}, N_{+}, N_{-}\right) / k_{B} T} \omega\left(N_{e}, N_{+}, N_{-}\right),
$$

where $\omega\left(N_{e}, N_{+}, N_{-}\right)$is the number of configurations of given $N_{e}, N_{+}$and $N_{-}$. We have calculated exact $\omega\left(N_{e}, N_{+}, N_{-}\right)$'s for all $N_{+}, N_{-}$for $N_{e}=0,1, \cdots, 6$. These $\omega$ 's are sufficient to calculate the partition functions at temperatures up to $k_{B} T / J=0.2$ for sizes up to $L=70$ with the machine precision. The phase diagram in $T-H-\Delta$ space looks like Fig. [ [9].

Defining order parameters, $\dot{N}=N_{+}+N_{-}$and $M=N_{+}-N_{-}$and their conjugate field variables, $\zeta=\beta\left(\Delta-\Delta_{o}\right)$ and $\eta=\beta\left(H-H_{o}\right)$, we can write the partition function as $Z(\zeta, \eta)=\sum_{\dot{N}, M} e^{-\zeta \dot{N}+\eta M} Z_{o}(\dot{N}, M)$. In the above $H_{o}$ and $\Delta_{o}$ are the values used to calculate the microcanonical partition function $Z_{o}(\dot{N}, M)$ which is defined as $Z_{o}(\dot{N}, M)=$ $e^{-\beta \Delta_{o} \dot{N}+\beta H_{o} M} \sum_{N_{e}} e^{-\beta J N_{e}} \omega\left(N_{e},(\dot{N}+M) / 2,(\dot{N}-M) / 2\right)$. If $H_{o}$ and $\Delta_{o}$ are chosen to be the transition point, $\zeta$ and $\eta$ will vanish at the transition point.

In order to maximize the finite size effect, we demonstrate zeros at the highest allowable temperature, $\beta=5.0$ where the high-order cumulants are appreciable while the contributions to $Z_{o}(\dot{N}, M)$ from terms of $N_{e}$ higher than 6 are still negligible. The transition points are determined by equating the free energies of the coexisting phases for the system size $L \simeq 60$. We found the triple point at this temperature, $P_{T}=\left(\zeta_{o} \equiv \beta \Delta_{o}=-0.0000454657, \eta_{o} \equiv\right.$ 
$\left.\beta H_{o}=0\right)$ (Fig. 1(e)). A typical asymmetric transition point which we chose for the demonstration is $P_{A}=\left(\zeta_{o}=1, \eta_{o}=1.0000061392784119\right)$, a point like Fig. 1(f).

In Fig. 2. we display $\ln \left(Z_{o}\left(N_{+}+N_{-}, N_{+}-N_{-}\right)\right.$by the size of the filled circle in $\left(N_{+}, N_{-}\right)$ plane at various points in the phase diagram. It should be noted that in these figures $M$ and $\dot{N}$ vary along the two diagonal lines.

We found that zeros in the complex $z_{\eta}\left(\equiv e^{\eta}\right)$-plane at phase points like Fig. 1(a) along the $\eta_{o}=0$ line up to the triple point are distributed more or less uniformly on the unit circle for all $L$ as predicted by the theorem. On the other hand there are no zeros along the zero-field line in $z_{\zeta}\left(\equiv e^{\zeta}\right)$-plane in the direction parallel to $\eta_{o}=0$ line as long as the phase points remain far away from the triple point. At $P_{T}$ we found zeros in $z_{\zeta}$-plane in the direction indicated by Fig. 1(e). form a circle of varying radius which is dependent on the size of the system as shown in Fig. 3A. Since the transition at $P_{T}$ in the direction parallel to $\eta_{o}=0$ line is asymmetric, it might be concluded that the partition functions of the two phases are added up with unequal prefactors. However the asymmetry in this case is due to the fact that it is a transition from the two-phase region to the single phase region, and the asymmetry factor $a$ in Eq.(3) is indeed exactly 2 if we fit the concentric circles by the predicted relations, $r=\exp \left[\ln (a) /\left(2 L^{d} \mu\right)\right]$.

On the other hand in the $z_{\eta}$-plane the uniformly distributed $2 N$ zeros pair up and converge to each other as $\zeta_{o}$ approach the triple point and they eventually double up before they bifurcate into two sets of concentric circles of $N$ zeros each at $P_{T}$. Fig. $3 \mathrm{~B}$ is the plot of zeros at $P_{T}^{\prime}=\left(\zeta_{o}=0.01088, \eta_{o}=0\right)$, a point slightly to the right of Fig. 1(b). The size dependence in this plot is not from the $L$ 's in $\tilde{F}(h)$ in Eqs. (9) and (10) which would give converging circles rather than divergence. The origin of the divergence is the $L$-dependence of the the parameter $b$ of Eq.(9). Since the phase point $P_{T}^{\prime}$ is far enough from the triple point so that $b(L) \propto \exp \left(\left(\zeta_{o}\left(P_{T}^{\prime}\right)-\zeta_{o}\left(P_{T}\right)\right) L^{d}\right), r$ diverges as $r \propto \exp \left(\left(\zeta_{o}\left(P_{T}^{\prime}\right)-\zeta_{o}\left(P_{T}\right)\right) L^{d}\right)$. Since circles of both sets converge to the unit circles if we move $\eta_{o}$ from $\eta_{o}=0$, to a coexistence point on either $B+$ or $B$ - plane of Fig. 1, the two sets are actually finite-size ghost circles that separate three phases bounded by $B+$ and $B$ - plane. 
Fig. $3 \mathrm{C}$ is a plot of zeros in $z_{\zeta}$-plane at $P_{A}$ and at two nearby points, $P_{A}^{ \pm}=\left(\zeta_{o}=\right.$ $\left.\zeta_{o}\left(P_{A}\right) \pm 0.000005, \eta_{o}=\eta_{o}\left(P_{A}\right)\right)$ Although zeros for only two sizes, $L=8$ and $L=40$ are shown, zeros of all sizes collapse into single curves. This proves that $a=1$, which implies that the partition functions of two phases are added up without a prefactor.

Fig. 3D is the plot of exact zeros at $P_{A}$, together with approximate zeros calculated using Eqs.(5) and (蛋). We calculated $\hat{\gamma}_{l}$ for $l=1$ through 6 and approximate zeros of successive order which include $\hat{\gamma}_{l}$ of successively higher orders. The reason that we see only 4 sets of approximate zeros is because $a=1$ or $\hat{R}=0$ makes the third and fifth terms of Eq.(四) vanish. It should be noted that $\hat{R}=0$ makes even-order terms of Eq.(5) vanish instead. This again proves $a=1$. It also shows that the locus of zeros for large $\theta$ is affected more and more by the discontinuities of higher order derivatives of the free energy, $\tilde{\gamma}_{l}$ 's as predicted by the theorem.

In conclusion we have shown that the extended circle theorem for first-order transitions follows from a very simple property that the distribution function for the order parameter is multiply peaked at the coexistence point. In fact zeros of the partition function can be expressed in terms of the position and shape of these peaks through cumulants of each phase. We have also found the finite-size effect on the zeros of the partition function which can be useful in determining the order of transition as well as the accurate estimate of the transition point from the numerical data of finite-size systems which can be easily obtainable from a Monte Carlo technique of Ref. [10] for example. Finally we have found that the partition functions are added up without a prefactor in the case of asymmetric phase transition in the Blume-Capel model, at least at low temperatures.

This work was supported in part by the Ministry of Education, Republic of Korea through a grant to the Research Institute for Basic Sciences, Seoul National University, in part by the Korea Science Foundation through Research Grant to the Center for Theoretical Physics, Seoul National University and in part by S.N.U. Daewoo Research Fund. The author wishes to thank Moo Young Choi and Julian Lee for the critical reading of the paper. 


\section{REFERENCES}

[1] C.N. Yang and T.D. Lee, Phys. Rev. 87, 404 (1952); T.D. Lee and C.N. Yang, Phys. Rev. 87, 410 (1952)

[2] R. B. Griffiths in Phase Transitions and Critical Phenomena vol. 1 ed. C. Domb and M.S. Green (Academic,New York, 1973) p 8

[3] K-C. Lee, Phys Rev. E 483459 (1993)

[4] K. Binder and D.P. Landau Phys Rev. B 30, 1477 (1984)

[5] M.S. Challa, D.P. Landau, and K. Binder, Phys Rev. B 34, 1481 (1986)

[6] C. Borgs and R. Kotecky, J. of Stat. Phys. 61, 79 (1990)

[7] M. Blume, Phys Rev. 141, 517 (1966)

[8] H. W. Capel, Physica. 32, 966 (1966)

[9] See e.g., R.B. Griffiths, Phys Rev. Lett. 30, 715 (1970)

[10] K-C Lee, J. Phys. A 23, 2087 (1990) 


\section{FIGURES}

FIG. 1. Schematic phase diagram of Blume-Capel model. The symmetric coexistence plane $A$ lies in $T-\Delta$ plane bounded by the triple line $O P$ and the critical line $P R$. Two asymmetric coexistence planes $B+$ and $B$ - bifurcate from the triple line symmetrically. $P$ is the tricritical point.

FIG. $2 . \ln \left(Z_{o}\left(\left(N_{+}+N_{-}, N_{+}-N_{-}\right)\right)\right.$on $\left(N_{+}, N_{-}\right)$plane for $L=16$. I. At $\zeta_{o}=-1, \eta_{o}=0$, a symmetric two-phase transition point corresponding to Fig. 1(a). II. At $P_{T}$. III. At $\zeta_{o}=1, \eta_{o}=0$, a symmetric single phase point far away from the triple point corresponding to Fig. 1(c). IV. At $P_{A}$.

FIG. 3. Zeros of partition function. Circles of a solid line are unit circles in all 4 figures. A. 6 sets of $N\left(=L^{2}\right)$ zeros for the system size, $L=8,10,12,14,16$ and $L=60$ in decreasing size of dots at $P_{T}$ in $z_{\zeta}$-plane. The radial distance from the unit circle is magnified 50 times. Even at this magnification zeros for $L=60$ is too close to the unit circle to be discernible. B. Zeros $P_{T}^{\prime}$ in $z_{\eta}$-plane for $L=8,10,12$ and $L=14$. Distance from the unit circle for outer circles is scaled down by a factor of $1 / 25$ to bring them into the plotting area. C. Zeros in $z_{\zeta}$-plane at $P_{A}$ (the circle

tangent to the unit circle), $P_{A}^{-}$(the one inside of the unit circle) and $P_{A}^{+}$(the largest circle which crosses the unit circle). In $\mathrm{C}$ and $\mathrm{D}$ the radial distance from the unit circle are again magnified this time 50000 times! D. Exact and approximate zeros at $P_{A}$. Zeros for two sizes, $L=8$ and 40 are shown. The unit circle is the zeroth order approximation, the innermost is the second order approximation, zeros closest to the unit circle is the fourth, and the sixth is closest to the exact zeros, the circle in the middle. Similar plots for zeros in $z_{\eta}$-plane (in the direction of Fig. 1(d)) are obtained at this point. 